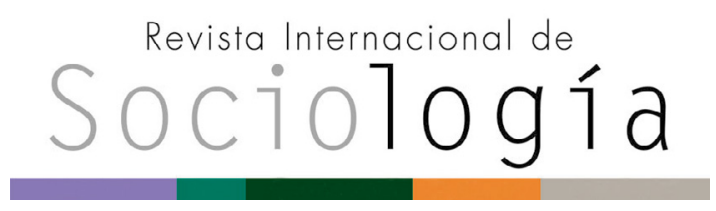

Revista Internacional de Sociología RIS

vol. 79 (1), e177, enero-marzo, 2021, ISSN-L:0034-9712 https://doi.org/10.3989/ris.2021.79.1.19.045

\section{SATISFACCIÓN CON LA VIDA SEGÚN LA CONTRIBUCIÓN A LA ESFERA PÚBLICA Y PRIVADA EN LAS PAREJAS ESPAÑOLAS ADULTAS}

\author{
JORDI GUMÀ \\ Universitat Pompeu Fabra y \\ jordi.guma@upf.edu \\ ORCID iD: https://orcid.org/0000-0003-2640-5391 \\ Bruno ARpino \\ Università degli Studi di Firenze \\ bruno.arpino@unifi.it \\ ORCID iD: http://orcid.org/0000-0002-8374-3066
}

Cómo citar este artículo / Citation: Jordi Gumà y Bruno Arpino. 2021. "Satisfacción con la vida según la contribución a la esfera pública y privada en las parejas españolas adultas". Revista Internacional de Sociología 79(1):e177. https://doi.org/10.3989/ ris.2021.79.1.19.045

\section{REsumen}

El objetivo del trabajo es, tomando como referencia la revolución de género, explorar si la desigual participación relativa en la provisión de recursos económicos y la realización de las tareas domésticas en el hogar se asocia con diferencias en los niveles de satisfacción con la vida para las mujeres y hombres adultos que conviven en pareja en España. Para ello, se analizan mediante modelos de regresión lineal las muestras españolas para la Encuesta Social Europea de 2004 y 2010 conjuntamente.

En España se observan dos situaciones diferentes definidas por la distinta capacidad económica del hogar: en los hogares sin dificultades económicas, los comportamientos de género tienen una menor influencia sobre los niveles de satisfacción con la vida; sin embargo, en aquellos con dificultades económicas los perfiles de género más tradicionales se asocian con una mayor satisfacción masculina, al contrario que en el caso femenino.

\section{Palabras clave}

Revolución de género; satisfacción con la vida; parejas; población adulta; España.

\section{LIFE SATISFACTION ACCORD- ING TO INDIVIDUAL CONTRIBU- TION WITHIN THE PUBLIC AND THE PRIVATE SPHERES AMONG PARTNERED SPANISH ADULT POPULATION}

Copyright: (c) 2021 CSIC. Este es un artículo de acceso abierto distribuido bajo los términos de la licencia de uso y distribución Creative Commons Reconocimiento 4.0 Internacional (CC BY 4.0).

Recibido: 26/03/2019. Aceptado: 25/05/2020 Publicado: 06/04/2021

\section{Abstract}

Our goal is, taking the gender revolution as a reference, to explore whether the relative contribution to the economy of the household and to carry out the domestic tasks of the household are associated with the life satisfaction of adult Spanish women and men seaparately who live with a partner. For this purpose we use linear regression models to analyze the data from the pooled Spanish samples of the European Social Surveys of 2004 and 2010.

In Spain there are two different situations defined by the different economic capacity of the household: in households without economic difficulties, g ender behaviors have a lower influence on levels of satisfaction with life; however, in those housholds with economic difficulties the more traditional gender profiles are associated with a greater male satisfaction, unlike in the female case.

\section{KEYWORDS}

Gender revolution; Life satisfaction; Couples; Adult population; Spain. 
Este trabajo forma parte del Proyecto "Más vale prevenir que curar cuando el envejecimiento esta detras de la puerta: interacción entre determinantes sociales de la salud en España (INTERSOC-HEALTH)" (RTI2018-099875-J-I00 -MCIU/AEI/FEDER, UE- PI: Jordi Gumà) financiado por el FEDER/Ministerio de Ciencia, Innovación y Universidad/Agencia Estatal de Investigación.

\section{INTRODUCCIÓN}

La ruptura con los modelos de género tradicionales en pos de un horizonte final de plena igualdad social entre mujeres y hombres, reivindicado largamente por parte del movimiento feminista, resultó ser el motor del cambio que empezó a observarse en el s. XX en las sociedades occidentales (Freedman 2007). Tomando como marco conceptual el propuesto por la revolución de género (traducción del inglés de la Gender revolution), se puede fijar el inicio del proceso de cambio a gran escala en el momento en que las mujeres empezaron a ganar visibilidad en la esfera pública (Stanfors y Goldscheider 2017). De este modo, de manera progresiva el modelo tradicional, en el cual el hombre se centraba en las tareas de proveedor de recursos mientras que la mujer debía dedicarse exclusivamente a las tareas domésticas, ha dejado de ser predominante, repercutiendo en una mayor heterogeneidad en los perfiles de pareja en cuanto a los niveles de igualdad de género dentro del hogar.

Este proceso de transformación social se ha observado a lo largo de las últimas décadas con desigual inicio e intensidad en los diferentes países europeos (Goldscheider Bernhardt y Lappegård 2015; Lewis, Campbell y Huerta 2008). Mientras que en los países del norte del continente este proceso de cambio hacia la igualdad social entre mujeres y hombres se encuentra en un estado más avanzado (Esping-Andersen y Billari 2015), en otros países este horizonte de igualdad aún parece lejano, debido a que el proceso de cambio empezó más tarde y, por tanto, se encuentra en una fase más temprana (Sayer 2015; Lewis, Campbell y Huerta 2008).

En el caso concreto de España, el país ha experimentado grandes y a la vez rápidos cambios sociales en esta dirección, aunque se debe tener en cuenta que partía de una situación de mayor desigualdad de género que la mayoría de países europeos, como consecuencia de la dictadura nacionalcatólica de Franco (1939-1975), que esteriotipó el rol de las mujeres en su papel de cuidadoras (Alonso-Pérez y Furió-Blasco 2007). A pesar de la rapidez de los cambios hacia mayores cotas de igualdad de género, este proceso se frena en el contexto de la reciente recesión económica, tanto en la esfera pública como en la privada, subrayando la importancia que tiene el contexto económico en la evolución de los niveles de desigualdad de género entre la población española (Murillo-Huertas y Simón-Pérez 2014; Lombardo y León 2014).

Existen númerosos trabajos que han analizado las consecuencias que los cambios en los roles de género han demostrado tener sobre factores demográficos como la fecundidad (Bueno y Brinton 2019; Arpino, Esping-Andersen y Pessin 2015), el matrimonio y el divorcio (Sassler y Lichter 2020; Pessin 2018). Sin embargo, se ha prestado una atención menor a cómo esta revolución de género se asocia con factores relacionados con el bienestar individual. En otras palabras, se tiene muy poco conocimiento sobre cómo se está viendo afectado el bienestar de las personas como consecuencia de los cambios en la esfera pública y privada.

Teniendo en cuenta el componente de transmisión intergeneracional de los comportamientos de género y las normas sociales en general (Billari y Liefbroer 2007), nuestra hipótesis de trabajo es que el hecho de que en España los cambios en la esfera pública se hayan iniciado de manera relativamente reciente implica que aún no ha dado tiempo a un remplazo general de las normas de género tradicionales que atañen a la esfera privada. Esto llevaría a una situación de tensión entre el contexto y los valores heredados que podría afectar a los niveles de bienestar individuales. De hecho, existen evidencias para el caso concreto español sobre un desequilibrio entre los valores de género, mucho mas igualitarios que las prácticas finales que se observan (Prieto 2010; Valiente-Fernández 1997). Además, teniendo en cuenta el ya comentado efecto del contexto económico sobre las actitudes de género en el conjunto del país, nuestra segunda hipótesis es que este efecto del contexto sería también de importancia a nivel de hogar, por lo que, en los hogares con mayores dificultades económicas, la influencia de las prácticas de género sobre el bienestar sería mayor.

El objetivo del trabajo es explorar la asociación entre el nivel subjetivo de satisfacción con la vida y el grado de participación relativa de mujeres y hombres dentro del hogar (contribución relativa al total de ingresos en el hogar y a la realización de las tareas domésticas) entre la población española adulta que convive en pareja, según la capacidad económica del hogar. Se ha escogido la contribución a los ingresos para medir cuál de los dos miembros de la pareja es el proveedor principal, más allá del hecho de trabajar o no. En cuanto a la realización de las tareas del hogar, permite aproximar si la repartición de estas tareas es equitativa o si, por el contrario, es responsabilidad de uno de los miembros de la pareja, que normalmente es la mujer.

La contribución del trabajo a la literatura actual es doble. Primero, se tiene en cuenta la asociación de la satisfacción con la vida, tanto con el papel de 
proveedor económico (esfera pública) como con la repartición de las tareas domésticas (esfera privada) de manera separada. El hecho de realizar los análisis de manera separada se debe a la intención de captar los efectos sobre la satisfacción con la vida en relación con ambas esferas de manera diferenciada, ya que el inicio del cambio en ambas esferas es distinto. Segundo, se incorpora la capacidad económica del hogar como un posible factor diferenciador a la vista de las evidencias encontradas en trabajos anteriores. Para ello, se analizan mediante modelos de regresión lineal las muestras españolas para la Encuesta Social Europea (ESS) de 2004 y 2010 conjuntamente, años en los cuáles se introdujeron las preguntas del módulo sobre familia, trabajo y bienestar. Los diferentes análisis se realizan de manera independiente según el sexo, para poder identificar posibles diferencias entre mujeres y hombres.

\section{Antecedentes}

El marco de referencia de nuestro trabajo es el de la "revolución de género" (Esping-Andersen y Billari 2015; Goldscheider Bernhardt y Lappegård 2015), en el cual se describen las dos etapas diferenciadas que caracterizan la evolución del proceso de cambio en los comportamientos de género en los países occidentales. En la primera de estas etapas, se observa que las mujeres poco a poco se muestran más visibles en la esfera pública como consecuencia, por ejemplo, de su mayor participación en el mercado laboral o de la progresiva asunción de puestos de gran visibilidad en la Administración Pública. Sin embargo, en esta primera etapa aún se observa la persistencia de los valores más tradicionales en el ámbito de la esfera privada, tal y como refleja el hecho de que la realización de los trabajos domésticos y de cuidado dentro del ámbito del hogar sigue siendo responsabilidad de las mujeres casi en su totalidad. Posteriormente, el proceso de cambio en la esfera pública empieza a tener calado en la esfera privada, produciéndose un incremento relativo de la población que adopta una mayor igualdad en la repartición de las tareas domésticas y de cuidado de los hijos e hijas, yendo, ahora sí, ambas esferas en la misma dirección.

Uno de los factores de mayor relevancia en el devenir de la primera etapa de la revolución de género fue la expansión educativa femenina, que llevó a que las mujeres pasaran de tener una participación casi testimonial en la educación superior a superar a los hombres a partir de finales del pasado siglo (Lutz 2010). Esto, unido al cambio de modelo económico en que el sector terciario ha ganado importancia por encima de los sectores de producción o agrícolas, facilitó que las reivindicaciones realizadas por parte del movimiento femenista durante muchas décadas, de una mayor igualdad de género, empezaran a dar resultados y se iniciara la primera etapa de la revolución (Stanfors y Goldscheider 2017).

Sin embargo, dentro del ámbito doméstico las mujeres seguían siendo las que, desde edades tempranas, eran preparadas por parte de las generaciones femeninas mayores para llevar a cabo las tareas del hogar. Esto se debe en parte al hecho de que, tal y como pasa con el resto de comportamientos y valores sociales, las convenciones de género dentro de la esfera privada se transmiten de generación en generación (Billari y Liefbroer 2007), lo cual ralentiza la velocidad de cambio. De hecho, incluso actualmente se siguen observando entre las mujeres comportamientos tradicionales como, por ejemplo, el hecho de que el potencial como proveedor económico del hombre siga siendo un factor relevante dentro del mercado matrimonial para un porcentaje significativo de las mujeres estadounidenses (Gerson 2010). Esta misma preferencia por parte de las mujeres en la elección de pareja fue observada en un estudio realizado entre estudiantes universitarias en España (Maté y Acarín 2011).

De manera general, las mujeres y los hombres tienen un protagonismo diferente en cada una de estas etapas. Mientras que la primera etapa se caracteriza por tener a las mujeres como motor del cambio en busca de un mayor protagonismo en la esfera pública, la segunda se produce cuando los hombres pasan a dedicar más tiempo a la esfera privada, aunque con una mayor resistencia por parte de ellos a perder su posición dominante en el contexto social. Por tanto, se observa que la generalización de la incorporación de la mujer al mercado laboral remunerado se está produciendo de manera más rápida que la asunción por parte de los hombres de una repartición equitativa de las tareas no remuneradas dentro del ámbito doméstico (Cherlin 2016; England 2010).

Las razones que explican esta diferencia son diversas. Por ejemplo, mientras que las mujeres pioneras en incorporarse al mercado laboral fueron acusadas de poner en peligro a sus familias, sin embargo nunca se les atribuyó la puesta en riesgo de la estabilidad económica de sus hogares. Por el contrario, los hombres en contadas ocasiones han tenido motivaciones de naturaleza económica que les lleven a adoptar una actitud más proactiva para contribuir en aquellos trabajos relacionados con la dinámica del hogar, como las tareas domésticas o el cuidado de sus hijos e hijas (Haas 1992). De hecho, son precisamente las motivaciones económicas, como, por ejemplo, el permiso de paternidad remunerado, las que han mostrado tener un mayor efecto positivo en el deseo de los padres de involucrarse en mayor medida en el cuidado de sus hijos e hijas (Appelbaum y Milkman 2013).

El resultante desequilibrio entre las esferas pública y privada ha mostrado hacer mella en el bienes- 
tar femenino como consecuencia de la sobrecarga derivada de tener que compaginar los trabajos de tipo remunerado y no remunerado (Rosales 2003). Sin embargo, esta sobrecarga ha mostrado tener un efecto desigual sobre un factor meramente subjetivo como la felicidad dependiendo de las nomas de género a nivel contextual. Mencarini y Sironi (2010) mostraron que los niveles generales de desigualdad de género observados en un país modificaban la magnitud del efecto que tenía la repartición de las tareas domésticas en el ámbito del hogar sobre los niveles de felicidad de las mujeres europeas. De este modo, la mayor carga femenina de trabajo doméstico y de cuidado demuestra tener un mayor efecto negativo sobre la felicidad en los países con menores niveles de desigualdad, mientras que en aquellos más desiguales el efecto negativo era menor.

No obstante, menos se sabe sobre los efectos que tiene esta repartición (equitativa o no) de las tareas en el seno del hogar sobre la felicidad de los hombres. En el caso español, aunque paradójicamente son una minoría los hombres que declaran tener que compaginar los trabajos remunerados y no remunerados, esta situación ha mostrado erosionar en mayor medida su calidad de vida, relacionada con la salud, que en el caso de sus homólogas femeninas (Gumà, Treviño y Cámara 2015). Los autores explicaron esta paradoja a partir del hecho de que los hombres expuestos a esta doble carga aún son una minoría, motivo por el cual valoran de manera más negativa esta situación cuando se comparan con el resto de los hombres. Esta explicación iría en el sentido de lo propuesto por Mencarini y Sironi (2010) en el caso de las mujeres, aunque en el de los hombres los mayores niveles de desigualdad de genero globales tendrían un efecto de incremento en la magnitud sobre este efecto negativo. A su vez, existen evidencias del efecto que sobre la salud mental masculina tiene el cambio en el modelo del hombre como proveedor principal de recursos económicos. Se ha demostrado que dejar de ser los principales proveedores de recursos económicos dentro del hogar socava sus niveles de autoestima en el conjunto del continente europeo, lo cual ha incidido en sus niveles de salud mental (Van der Meer 2014).

Aunque la revolución de género en el contexo de los países occidentales presenta características muy similares, se observa que la transición entre las dos etapas tiene ritmos diferentes en diferentes regiones del continente europeo. Los países del norte de Europa fueron los primeros en los cuales se pudo observar esta revolución, seguidos por los países de la parte central del continente (Aassve, Fuochi y Mencarini 2014; Brinton y Lee 2013). Por el contrario, este proceso se muestra más lento en los países del sur de Europa, donde los valores tradicionales de relación entre mujeres y hombres aún se dan en un porcentaje relativamente alto de la población
(Brinton y Lee 2013; Moreno-Mínguez 2010). Entre estos países, España resulta ser un caso de gran interés por el relativamente rápido incremento de la participación de las mujeres en la esfera pública observado en las últimas décadas gracias, en parte, al desarrollo de las políticas en pro de la igualdad de género llevadas a cabo desde principios de la democracia (Lombardo y León 2014), aunque hay que tener en cuenta que el periodo de la dictadura hizo que el punto de partida en cuestiones de género fuera realmente bajo. A lo largo del periodo dictatorial hubo muy poco margen para ir más allá de las normas tradicionales de género, e incluso durante los años de fuerte crecimiento económico del periodo desarrollista del franquismo (1963-75) se observó que aumentaba la desigualdad salarial entre mujeres y hombres (Martín y Sánchez-Sánchez 2007).

En la actualidad, España sigue mostrando indicios de un progresivo cambio hacia una mayor igualdad entre mujeres y hombres dentro de la esfera privada, tal y como muestra el análisis sobre el uso del tiempo de los miembros de las parejas. Cuando se comparan los datos entre los períodos 2002-2003 y 2009-10, se observa un incremento del porcentaje de parejas en las cuales la mujer participa en el trabajo remunerado (Ajenjo y García 2014). Así mismo, entre estos dos periodos se puede ver un incremento en el tiempo que los hombres emplean en el cuidado de sus hijos e hijas menores de 12 años (Borràs, Ajenjo y Moreno-Colom 2018).

Sin embargo, estos prometedores resultados contrastan con la posición del país dentro del contexto europeo. En el año 2017, España seguía mostrando peores valores a nivel agregado que la media de la UE 28 en indicadores como la diferencia entre el empleo masculino y el femenino o el porcentaje de mujeres en puestos de responsabilidad (Eurostat 2018). Además, el interés del caso español radica en el desequilibrio existente entre los valores y las prácticas. Los resultados muestran que mientras la mayoría de la población española opina que las tareas del hogar se deben compartir por igual entre ambos miembros de la pareja, el porcentaje de parejas españolas en las cuales se lleva a la práctica esta idea es mucho menor que en otros países occidentales (Prieto 2010; Valiente-Fernández 1997). Esta paradoja sería síntoma del desequilibrio actual entre los cambios en cuestiones de género que se han producido en la esfera pública y los que aún deben darse en la esfera privada.

Es precisamente esta situación de desequilibrio la que subraya el interés de estudiar cómo los diferentes balances de género dentro del hogar afectan a la satisfacción con la vida de las personas, sobre todo si asumimos que el país parece no encontrarse en una situación avanzada en ninguna de las dos etapas de la revolución de género (e.g., menor par- 
ticipación femenina en el mercado laboral respecto a la media de la Unión Europea - 60 \% mujeres actvias en España vs. 66,5 \% de la UE en 2017, según EUROSTAT 2018-; menor participación de los hombres en las tareas domésticas, etc.).

\section{Metodología Y FUENTE dE dATOS}

Los datos utilizados en este trabajo provienen de las muestras españolas de la European Social Survey (ESS) para los años 2004 y 2010, correspondientes a la segunda y quinta ediciones, respectivamente. Esta encuesta se realizó por primera vez en el año 2002 y, desde entonces, se repite cada dos años en la casi totalidad de países europeos (más Israel), entrevistando en cada edición a personas diferentes para garantizar la independencia entre muestras. El proceso de muestreo en cada edición se basa en métodos probabilísticos de selección de hogares e individuos con el objetivo de conseguir muestras independientes representativas de la población de 15 años y más para cada país y año de encuesta (Fitzgerald y Jowell 2010). La calidad del proceso de muestreo en los diferentes países se ha validado mediante la comparación con otra fuente estadística de calidad contrastada como es la Labour Force Survey realizada por EUROSTAT (Koch et al. 2014).

El cuestionario de la ESS consta de dos partes: un conjunto de preguntas troncales que se repiten en cada una de las ediciones, y uno o más módulos diferentes de cuestiones específicas que se alternan en las diferentes ediciones de la encuesta. La elección de las oleadas utilizadas en nuestro trabajo se debe a que, en estas ediciones, se incluyeron preguntas específicas sobre los temas que se encuentran en el foco de nuestra investigación: familia, trabajo y bienestar. La muestra de trabajo se ha restringido a las personas que declararon convivir en pareja y cuyas edades están dentro del rango 25-59, con el objetivo de trabajar con la muestra de población adulta en edad laboral. La justificación de la elección de este rango de edades específico se basa en la intención de reducir el posible sesgo en nuestros resultados, resultante del hecho de trabajar con edades demasiado jóvenes para haber finalizado estudios superiores (razón por la cual la edad mínima se fija en 25), o de incluir personas ya jubiladas (la edad media efectiva de la jubilación en el periodo 2013-2018 fue de 62.1 y 61.3 para hombres y mujeres respectivamente, según la OECD 2018).

Se analizaron los datos para las ediciones de 2004 y 2010 de manera conjunta, debido a la proximidad temporal de ambas ediciones, con el objetivo de incrementar la muestra específica para España $y$, de este modo, aumentar la capacidad para obtener resultados robustos desde el punto de vista es- tadístico. Previamente se comprobó que no existían diferencias significativas en los resultados entre ambos años, tanto de manera descriptiva (la tabla A1 muestra igualdad en el perfil de las muestras para ambos años, siendo la capacidad económica del hogar la única excepción) como multivariable (resultados no significativos para la variable "Año" en los modelos multivariables de las tablas $\mathrm{A} 2$ y $\mathrm{A} 3$ ) para las muestras españolas. De este modo, la muestra inicial del trabajo ha sido de 1512 personas, de las cuales finalmente se obtuvo información para todas las variables de nuestro análisis en 1440 casos (53 $\%$ de mujeres).

\section{Variable dependiente}

\section{Satisfacción con la vida}

Para aproximar el bienestar subjetivo de las personas entrevistadas se ha optado por trabajar con la variable de satisfacción con la vida actual. La pregunta concreta que aparece en el cuestionario de la encuesta es la siguiente: "en términos generales, ¿en qué medida está Ud. satisfecho con su vida actualmente?". Este ítem recoge la valoración subjetiva de la satisfacción de las personas en general, más allá de cuestiones como el bienestar físico o la coyuntura económica del momento. De manera formal, se trata de una escala de tipo Likert, tomando valores entre 0 (totalmente insatisfecho/a) y 10 (totalmente satisfecho/a).

\section{Variables independientes de interés}

\section{Contribución relativa a los ingresos del hogar}

Esta variable mide la contribución relativa de cada persona al presupuesto del hogar, identificando de este modo si esta persona es el principal proveedor o no. El literal de la pregunta fue: “¿qué proporción de los ingresos de este hogar aporta Ud.?", incluida en el cuestionario de las dos ecidiones de la ESS estudiadas. Las respuestas finales fueron agrupadas en tres categorías finales: menos de la mitad (ninguna, una pequeña parte y menos de la mitad); aproximadamente la mitad (aproximadamente la mitad); y más de la mitad (más de la mitad, una gran parte y todo).

\section{Contribución relativa a la realización de las tareas del hogar}

Esta variable mide la contribución relativa a la realización de las tareas cotidianas del hogar (tareas domésticas y cuidado de las personas dependientes) de la persona entrevistada respecto al total. En este caso, las variables originales no han podido ser las mismas en las dos ediciones de la ESS analizadas, debido al cambio de criterio establecido para medir el fenómeno, lo que ha hecho necesario buscar una solución de 
convergencia entre ambas ediciones. La edición de 2004 preguntó por la proporción del tiempo destinado a las tareas del hogar (nada, menos de un 1/4, entre un $1 / 4$ y $1 / 2$, más de $1 / 2$ y menos de $3 / 4$, más de $3 / 4$ y todo) asumido tanto por la persona entrevistada como por su pareja, diferenciando entre los días laborables y los fines de semana. De este modo, se pudieron calcular las tres categorías finales mediante la comparación de la información de la persona entrevistada y su pareja: la persona entrevistada dedicaba más tiempo que su pareja; dedicaba un tiempo equivalente al de su pareja, o destinaba menos tiempo que su pareja. Una vez comprobado que los resultados para los días laborables y para los fines de semana eran prácticamente los mismos, se optó por tomar la información para los días laborables, debido a que refleja mejor el equilibrio entre el tiempo destinado al trabajo remunerado y el no remunerado.

En cambio, en la edición de 2010 se preguntaba directamente por el número total de horas que tanto la persona entrevistada como su pareja dedicaban a lo largo de la semana completa a la realización de las tareas domésticas. Por lo tanto, para este año el cálculo de las nuevas tres categorías equivalentes a las calculadas para el año 2004 (la persona entrevistada dedica más, menos o igual tiempo a las tareas domésticas que su pareja) se pudo hacer de manera directa, estableciendo como criterio para la igualdad el hecho de que la diferencia entre las horas de ambos miembros de la pareja no sea superior a dos horas.

\section{Variables de control}

\section{Valoración subjetiva de la capacidad económica del hogar}

Esta variable se ha introducido en los análisis con el objetivo de tener en cuenta la posible influencia de la capacidad económica del hogar sobre la variable dependiente a través de su interacción con las dos variables de interés en nuestro trabajo. El literal original de la pregunta, que se repite en todas las ediciones de la ESS, es: " ¿cuál de las afirmaciones en esta tarjeta describe mejor cómo se siente con respecto a los ingresos de su hogar en la actualidad?". Las posibles respuestas han sido agrupadas del siguiente modo: cómodamente (con los ingresos actuales vivimos cómodamente); suficiente (con los ingresos actuales nos llega para vivir); con dificultades (con los ingresos actuales tenemos dificultades o muchas dificultades).

\section{Nivel educativo}

Las posibles respuestas a la pregunta sobre el máximo nivel de estudios finalizado han sido agrupadas de la siguiente manera, tomando como referencia los niveles de la clasificación general ISCED
(International Standard Classification of Education): nivel bajo (estudios de primer grado de secundaria o menor); nivel medio (segundo grado de secundaria y formación profesional superior), y nivel alto (estudios universitarios).

\section{Estado de salud}

Esta variable se ha introducido como control debido a su conocida influencia sobre la percepción del bienestar general de las personas. Con este objetivo, se ha tomado la evaluación subjetiva sobre el estado de salud en general que fue recogida mediante la respuesta a la pregunta: "hablando de su salud, en general, ¿diría usted que su salud es...?". Las posibles respuestas originales se han agrupado de la siguiente manera: buena salud (salud muy buena o buena); mala salud (salud regular, mala o muy mala).

\section{Edad}

Los modelos se controlan por edad debido también a su influencia sobre el bienestar subjetivo de la población. Esta variable se ha utilizado como discreta, tomando valores dentro del rango entre 25 y 59 . También se probó a añadir esta variable al cuadrado, aunque no mostró ser significativa, motivo por el cuál se decidió no incluirla en los análisis finales.

\section{Año}

Debido al hecho de trabajar con las muestras de dos ediciones diferentes de la ESS, se ha incluido una variable que controle por el año de la entrevista, 2004 o 2010.

El análisis estadístico realizado consta de dos partes. Una primera parte descriptiva en la cual se muestra para mujeres y hombres la distribución relativa de las diferentes variables analizadas en este trabajo. Además, dentro del apartado descriptivo se incluyen los valores promedio de satisfacción con la vida según las categorías de todas las variables, asi como el test correspondiente para evaluar las posibles diferencias en los promedios de las categorías dentro de las mismas variables. En la segunda parte, se muestran los resultados del análisis multivariable mediante regresión lineal para evaluar la asociación entre el nivel de satisfacción con la vida y la interacción entre la capacidad económica del hogar y las dos variables de interés en nuestro trabajo (contribución a los ingresos del hogar y a las tareas domésticas) por separado, controlando por las variables enumeradas en el apartado anterior.

El método escogido, el modelo de regresión lineal, ha sido previamente utilizado en numerosos trabajos para explorar la variabilidad de la satisfacción con la vida según factores de naturaleza tanto sociodemográfica como de salud (Arpino y de Valk 2018; Orru et al. 2016; Fernández-Alonso et al. 2012). Se han calculado modelos distintos para la interacción 
entre la capacidad económica del hogar y nuestras dos variables de interés (contribución a los ingresos y realización de las tareas del hogar) para obtener resultados representativos de cada esfera, teniendo en cuenta el calendario diferente en el inicio de los cambios en las esferas públicas y privadas.

Todos los análisis se han realizado de manera independiente para mujeres y hombres, con el objetivo de poder detectar posibles diferencias tanto en la magnitud como en el signo de los coeficientes. La comparación directa de la magnitud de los coeficientes es posible debido precisamente al hecho de trabajar con modelos de regresión lineal (Mood 2010).

Para facilitar la interpretación de los resultados, se ha optado por mostrar de manera gráfica la estimación de los valores resultantes para cada uno de los modelos según la variable de interés estudiada, la variable dependiente y el sexo de la persona entrevistada. Los coeficientes de los modelos pueden ser consultados en las tablas anexas A2 y A3. Todos los análisis se realizaron mediante el programa STATA (versión 14).

\section{Resultados}

\section{Análisis Descriptivo}

En la tabla 1 se puede ver que la muestra española de la ESS para las ediciones de 2004 y 2010 de población adulta (25-59) que convive en pareja sigue presentando un perfil desigual en cuestiones de género: un $77,7 \%$ de las mujeres dedican más tiempo que sus parejas a la realización de las tareas del hogar, mientras que un 73,4 \% de los hombres declaran ser los que aportan una mayor cantidad de dinero al presupuesto conjunto del hogar. La perecepción sobre la capacidad económica del hogar muestra valores casi iguales para mujeres y hombres, lo que apunta hacia una similar percepción sobre el contexto económico en ambos sexos. La mayoría de los hogares entrevistados llegan a final de mes o incluso lo hacen cómodamente (más de $78 \%$ en total en ambos sexos), mientras que un poco más del $20 \%$ declara tener dificultades económicas. En cuanto al nivel educativo, se observa un porcentaje mayor de

Tabla 1.

Características de la muestra de trabajo según sexo

\begin{tabular}{|c|c|c|c|}
\hline & & Mujeres & Hombres \\
\hline \multirow{4}{*}{ Capacidad económica del hogar } & Cómodamente & $34,8 \%$ & $36,8 \%$ \\
\hline & Llega a final de mes & $43,3 \%$ & $43,2 \%$ \\
\hline & Dificultades & $21,9 \%$ & $20,0 \%$ \\
\hline & Total & $100 \%$ & $100 \%$ \\
\hline \multirow{4}{*}{ Contribución relativa a los ingresos del hogar } & Menos de la mitad & $64,8 \%$ & $9,7 \%$ \\
\hline & Mitad & $23,7 \%$ & $17,6 \%$ \\
\hline & Más de la mitad & $11,5 \%$ & $72,8 \%$ \\
\hline & Total & $100 \%$ & $100 \%$ \\
\hline \multirow{4}{*}{ Contribución relativa a la realización de las tareas del hogar } & Menos que la pareja & $7,0 \%$ & $77,2 \%$ \\
\hline & Igual que la pareja & $15,3 \%$ & $14,5 \%$ \\
\hline & Más que la pareja & $77,7 \%$ & $8,3 \%$ \\
\hline & Total & $100 \%$ & $100 \%$ \\
\hline \multirow{3}{*}{ Salud } & Buena o muy buena & $65,0 \%$ & $73,4 \%$ \\
\hline & $\begin{array}{l}\text { Regular, mala o muy } \\
\text { mala }\end{array}$ & $35,1 \%$ & $26,6 \%$ \\
\hline & Total & $100 \%$ & $100 \%$ \\
\hline \multirow{4}{*}{ Nivel educativo } & Bajo & $54,0 \%$ & $48,3 \%$ \\
\hline & Medio & $21,7 \%$ & $24,5 \%$ \\
\hline & Alto & $24,3 \%$ & $27,2 \%$ \\
\hline & Total & $100 \%$ & $100 \%$ \\
\hline \multirow{3}{*}{ Año de la encuesta } & 2004 & $46,0 \%$ & $45,6 \%$ \\
\hline & 2010 & $54,0 \%$ & $54,4 \%$ \\
\hline & Total & $100 \%$ & $100 \%$ \\
\hline \multicolumn{2}{|l|}{ Edad media } & 42,5 & 43,3 \\
\hline \multicolumn{2}{|l|}{ Satisfacción con la vida } & 7,4 & 7,3 \\
\hline \multicolumn{2}{|l|}{$\mathbf{N}$} & 763 & 677 \\
\hline
\end{tabular}

Fuente: Muestras españolas ESS 2004 y 2010 
mujeres con nivel bajo respecto a los hombres (54\% vs. $48,3 \%$ ), mientras que, en el caso del nivel educativo alto, los valores son muy parecidos $(24,3 \%$ y $27,2 \%$ para mujeres y hombres, respectivamente). Esta pequeña diferencia aún a favor de los hombres se debería al hecho de que la muestra de trabajo contiene generaciones nacidas antes del proceso de expansión educativa que se ha observado en las últimas décadas en España.

También destaca el hecho de que los valores medios de satisfacción con la vida sean similares para mujeres y hombres. En cambio, en el caso de la salud se observa el ya conocido mejor perfil masculino respecto a las mujeres con igual perfil sociodemográfico (Oksuzyan et al. 2008). Por último, los valores de la edad media de mujeres y hombres son casi iguales, siendo ellos ligeramente mayores en promedio.

La tabla 2 muestra los valores promedio para mujeres y hombres sobre la satisfacción con la vida según todas las variables incluidas en los análisis, así como los resultados para los test estadísticos correspondientes para poder discernir si existen diferencias significativas entre los promedios de las diferentes categorías. Aunque los resultados se han calculado de manera separada para mujeres y hombres, los valores medios de satisfacción con la vida no eran significativamente diferentes en nuestra muestra de trabajo $(7,40$ y 7,39 para mujeres y hombres, respec- tivamente; $p$ valor para el t-test $=0,96)$. Sin embargo, sí que se observan diferencias según las diferentes variables, tal y como se muestra a continuación.

De manera general, se observa un patrón común para ambos sexos en el caso de la salud autopercibida, el nivel educativo y la capacidad económica del hogar. Estas tres variables muestran diferencias significativas entre sus categorías tanto en mujeres como en hombres, dejando ver un mismo patrón: mejor salud, mayor capacidad económica y nivel educativo más alto muestran mayores promedios de satisfacción con la vida. Cabe destacar que la diferencia que muestra una mayor significación estadística es la que se observa entre las categorías de la capacidad económica del hogar. También hay coincidencia en la igualdad de valores entre ambos años para la satisfacción con la vida, apuntando a que no hubo diferencias generales en cuanto a la variable dependiente de nuestro análisis entre las dos ediciones de la ESS.

Sin embargo, sí se observan diferencias precisamente en las dos variables de interés, la contribución relativa a los ingresos y la realización de tareas en el contexto del hogar. La primera se da en la signficación de las diferencias entre categorías de la misma variable. Estas diferencias son significativas entre los hombres, pero no entre las mujeres. La segunda diferencia se observa en el patrón de

Tabla 2.

Promedio de satisfacción con la vida según las características de la muestra de trabajo por sexo.

\begin{tabular}{|c|c|c|c|c|c|c|c|}
\hline & & \multicolumn{3}{|c|}{ Mujeres } & \multicolumn{3}{|c|}{ Hombres } \\
\hline & & Media & $\begin{array}{l}\text { Desviación } \\
\text { típica }\end{array}$ & Signif. & Media & $\begin{array}{l}\text { Desviación } \\
\text { típica }\end{array}$ & Signif. \\
\hline \multirow{2}{*}{ Salud autopercibida } & Buena & 7,63 & 1,5 & *** & 7,51 & 1,5 & ** \\
\hline & Mala & 6,99 & 2,1 & & 7,09 & 1,9 & \\
\hline \multirow{3}{*}{ Nivel educativo } & Bajo & 7,32 & 1,9 & $\dagger$ & 7,26 & 1,7 & * \\
\hline & Medio & 7,39 & 1,7 & & 7,42 & 1,6 & \\
\hline & Alto & 7,67 & 1,4 & & 7,63 & 1,3 & \\
\hline \multirow{3}{*}{$\begin{array}{l}\text { Contribución relativa a los ingresos } \\
\text { del hogar }\end{array}$} & Menos mitad & 7,42 & 1,8 & & 6,88 & 1,7 & * \\
\hline & Mitad & 7,46 & 1,6 & & 7,50 & 1,5 & \\
\hline & Más mitad & 7,21 & 1,8 & & 7,45 & 1,6 & \\
\hline \multirow{3}{*}{$\begin{array}{l}\text { Contribución relativa a la realización } \\
\text { de las tareas del hogar }\end{array}$} & Menos que la pareja & 7,13 & 1,8 & & 7,47 & 1,6 & $\dagger$ \\
\hline & Igual que la pareja & 7,46 & 1,8 & & 7,22 & 1,9 & \\
\hline & Más que la pareja & 7,43 & 1,7 & & 7,08 & 1,7 & \\
\hline \multirow{3}{*}{ Capacidad económica del hogar } & Cómodamente & 7,82 & 1,5 & *** & 7,78 & 1,3 & *** \\
\hline & Llega a final de mes & 7,40 & 1,6 & & 7,46 & 1,6 & \\
\hline & Dificultades & 6,76 & 2,2 & & 6,57 & 2,0 & \\
\hline \multirow{2}{*}{ Año } & 2004 & 7,41 & 1,8 & & 7,38 & 1,7 & \\
\hline & 2010 & 7,40 & 1,8 & & 7,41 & 1,6 & \\
\hline
\end{tabular}

Fuente: Muestras españolas ESS 2004 y 2010

Significación: ${ }^{* *}<0,001 ;{ }^{* *}<0,01 ;{ }^{*}<0,05 ; \dagger<0,1$

Nota: Para las variables con dos categorías (salud autopercibida y año) se ha utilizado el t test. En el resto de variables con más de dos se ha realizado mediante ANOVA. 
los valores promedio dentro de las dos variables. Las mujeres muestran promedios mayores de satisfacción con la vida cuando su contribución a los ingresos del hogar es la mitad o menos de la mitad, mientras que entre los hombres se observa el patrón inverso. Mientras que en el caso de la realización de las tareas domésticas, las mujeres muestran mayores promedios cuando declaran dedicar igual o más tiempo que sus parejas, mientras que los hombres de nuevo muestran un patrón inverso (mayor satisfacción cuanto menor es su aportación a la realización de estas tareas).

\section{Análisis Multivariable}

El grupo de figuras 1 muestra los marginales estimados del nivel de satisfacción con la vida para mujeres y hombres según la interacción entre la contribución al presupuesto del hogar y la capacidad económica del hogar, controlando por el nivel educativo, el estado de salud y el año de realización de la encuesta. Se observa que las grandes diferencias en los niveles de satisfacción con la vida se establecen invariablemente mediante la percepción sobre la capacidad económica del hogar en mujeres y hombres. Aquellas personas que perciben una mejor ca- pacidad económica en su hogar muestran mayores niveles de satisfacción con su vida. Únicamente en el caso de las personas que declaran tener dificultades económicas se observan diferencias significativas según su contribución relativa a los ingresos del hogar. Estas diferencias muestran un patrón inverso en mujeres y hombres. Mientras que ellas, cuando tienen dificultades económicas, muestran menores niveles de satisfacción con su vida cuanto mayor es su contribución al presupuesto del hogar, en el caso masculino es totalmente al contrario, siendo más altos sus niveles de satisfacción cuanto mayor es su contribución relativa.

En el caso de la contribución a la realización de las tareas del hogar (grupo de figuras 2), se observa que la capacidad económica no establece diferencias tan claras en cuanto a los niveles de satisfacción con la vida como en el caso de la contribución a los ingresos del hogar. Las únicas diferencias que se observan son en el caso femenino, cuando son ellas las que realizan la mayoría de las tareas domésticas en un contexto de dificultades económicas, mostrando valores menores que aquellas mujeres que también dedican más tiempo que sus parejas a estas tareas, pero tienen una mejor situación económica. En el caso de los hombres, la única diferencia significati-

\section{Grupo de Figuras 1.}

Estimación lineal de los valores de satisfacción con la vida según la contribución relativa a los ingresos del hogar por sexo

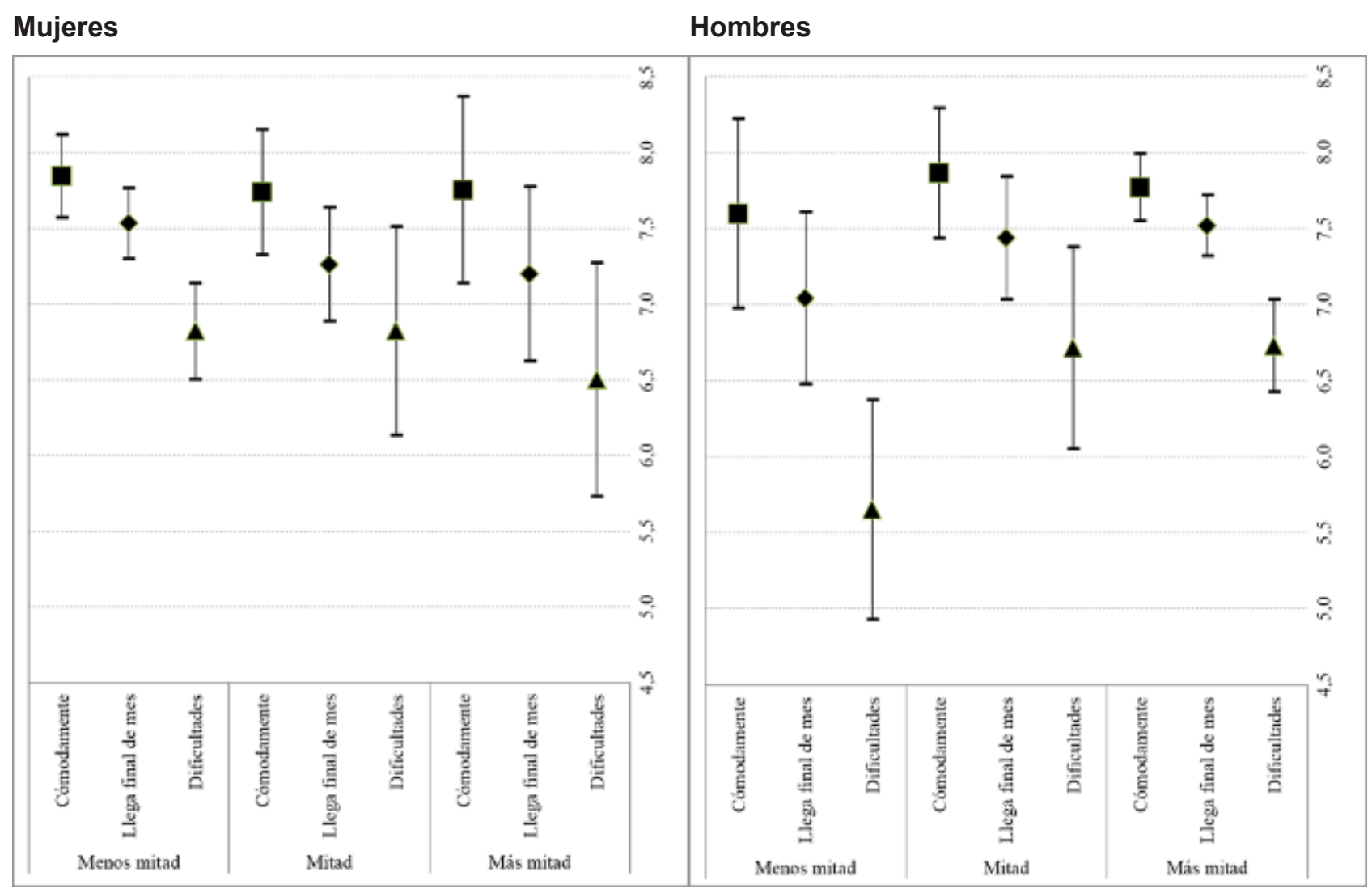

Fuente: Muestras españolas ESS 2004 y 2010 
Grupo de Figuras 2.

Estimación lineal de los valores de satisfacción con la vida según contribución relativa a la realización de las tareas del hogar por sexo

Mujeres

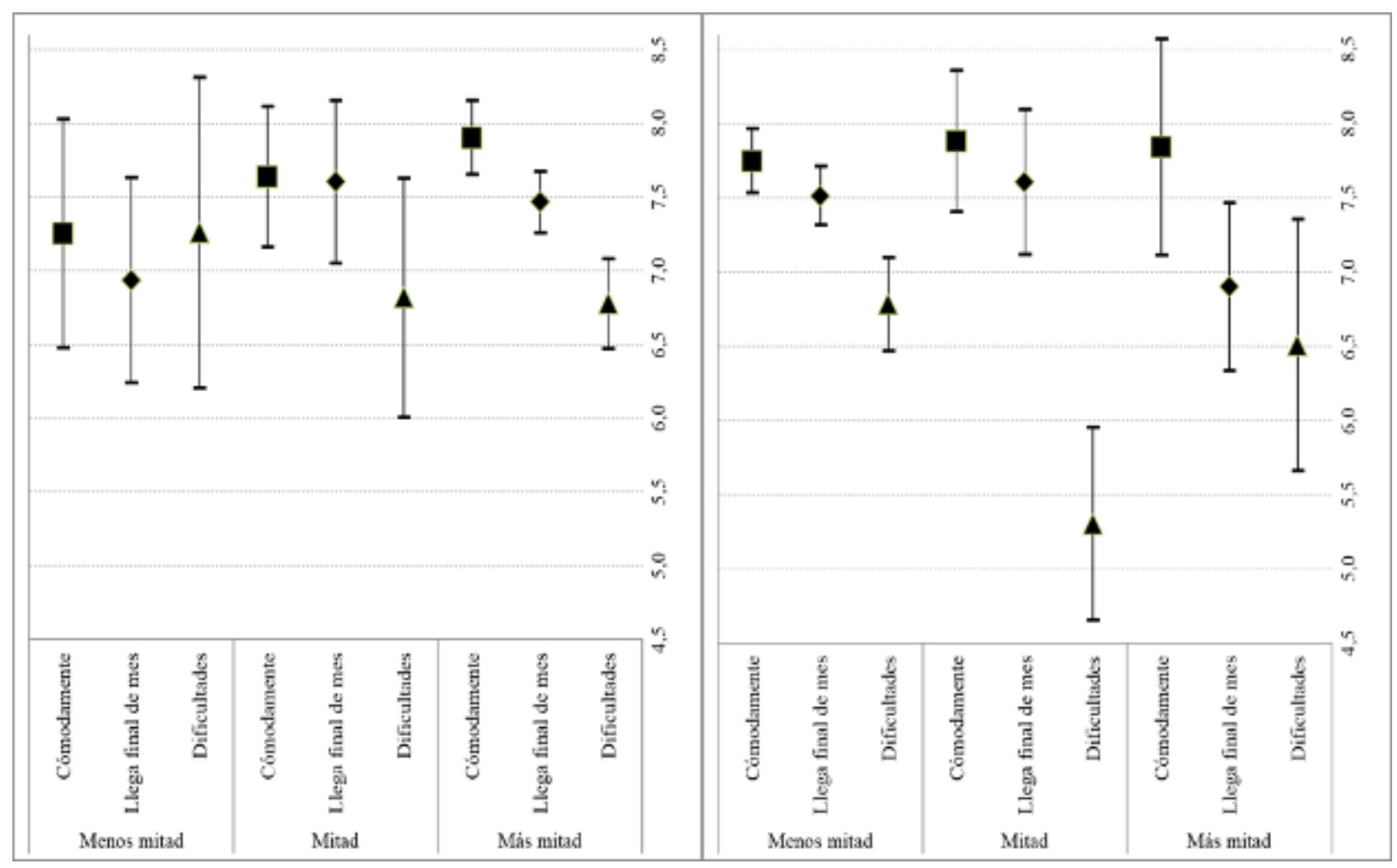

Fuente: Muestras españolas ESS 2004 y 2010

va se observa de nuevo en hogares con dificultades económicas, cuando ellos colaboran por igual en la realización de las tareas, mostrando también los valores más bajos de satisfacción con diferencia.

\section{Discusıón}

Este trabajo explora la asociación entre el nivel de satisfacción subjetivo con la vida y el grado de participación relativo de mujeres y hombres dentro del hogar, tanto en la provisión de recursos económicos como en la realización de las tareas domésticas, entre la población española adulta que convive en pareja. Los datos analizados proceden de las muestras españolas para la Encuesta Social Europea de 2004 y 2010 conjuntamente, de manera indpependiente para mujeres y hombres.

De manera general, el nivel de satisfacción con la vida parece estar marcado en gran parte por la capacidad económica del hogar. Este efecto por parte de la capacidad económica, con total probabilidad, se debe a la conjunción de diversos factores relacionados con la percepción subjetiva de las personas para evaluar cuán satisfechas están con la vida que han tenido y su situación en la actualidad. Esto pare- cería ser equiparable a la capacidad ya encontrada en trabajos anteriores sobre cómo el nivel educativo de las personas permite resumir los niveles de estratificación social en España (Martínez-Celorrio y Marín-Saldo 2012). Sin embargo, se debe recordar que precisamente la educación era una de las variables de control en los modelos calculados, lo cual lleva a pensar que ambas variables tienen un efecto complementario que permite entender con mayor precisión las diferencias en los niveles de satisfacción con la vida entre la población adulta española. En el caso español, la complementariedad entre ambas variables puede explicarse por la baja capacidad del mercado de trabajo para absorver en su mayoría los perfiles profesionales más formados, lo que demuestra una reducción de la capacidad de la educación como motor de promoción social, tal y como se apunta en el VIII Informe FOESSA (2019). Esto se ha traducido en un incremento progresivo desde 1980 de la incidencia relativa de la pobreza entre la población con estudios superiores, aunque estas personas sigan mostrando unos valores menores respecto a los niveles educativos más bajos. Se trata de un proceso que se está produciendo en un contexto de expansión educativa general en el país, que ha mostrado ser de especial importancia entre las mujeres espa- 
ñolas, las cuales actualmente muestran porcentajes de educación superiores más elevados que los hombres entre las generaciones más jóvenes (Gil-Hernández, Marqués-Perales y Fachelli 2017).

Sin embargo, más allá de la influencia general de la situación económica particular de cada hogar, se observa que los factores escogidos para aproximar los comportamientos de género en el contexto de las parejas españolas adultas, la contribución relativa a los ingresos del hogar y la realización de las tareas del hogar, tienen un efecto siginificativo sobre la satisfacción con la vida en el caso específico de las personas con dificultades económicas. Este efecto va en la dirección de las convenciones de género más tradicionales, aunque de manera diferente para mujeres y hombres.

Entre las mujeres, su satisfacción con la vida parece estar definida, sobre todo, por la situación global del hogar, más alla de su situación particular en cuanto su contribución relativa a los ingresos del hogar y al tiempo relativo dedicado a las tareas domésticas. De hecho, esta no es la primera vez que se encuentra que las mujeres tienden a dar mayor peso a las características del conjunto del hogar más allá de su situación particular cuando se analizan indicadores naturaleza subjetiva, como es el caso de la salud autopercibida, la calidad de vida (Doblhammer y Gumà 2018; Gumà, Treviño y Cámara 2015) o la satisfacción con la vida en el caso del presente trabajo. Por tanto, parece que es precisamente la situación dentro de la esfera privada la que sigue dibujando las diferencias en los valores de satisfacción con la vida entre las mujeres españolas.

A pesar de lo anterior, es digno de mención el diferente patrón encontrado en los valores de satisfacción femeninos según el tiempo dedicado a las tareas del hogar cuando interactua con la situación económica del hogar. Entre las mujeres que declaran no tener dificultades económicas (llegan a final de mes de manera suficiente o cómoda), se observa una relación positiva entre sus niveles de satisfacción y el tiempo dedicado a las tareas del hogar: cuanto mayor es su dedicación, mayor es su nivel de satisfacción. Sin embargo, este patrón es exactamente el contrario en el caso de las mujeres que declaran tener dificultades económicas, entre las cuales cuanto mayor es su dedicación a las tareas del hogar, menor su satisfacción. Este último punto reforzaría la idea de la importancia del conjunto del hogar sobre la satisfacción con la vida de las mujeres más allá de su situación individual, aunque se debería realizar un análisis más profundo en el futuro para entender las razones detrás de estos resultados. Una explicación posible es que aquellos hogares con una mayor capacidad económica tendrían una mayor capacidad de contratar servicios remunerados para realizar las tareas del hogar, por lo que, aunque estas mujeres dediquen más tiempo a las tareas del hogar que sus parejas, el número absoluto de horas destinadas puede ser menor que las que declaran tener dificultades económicas (en la muestra de 2010, la única para la cual se tiene esta información, las mujeres que llegan cómodamente a final de mes declaran dedicar 9 horas menos a la semana en promedio a las tareas del hogar que aquellas). Sin embargo, este menor número de horas no implica encontrarse en un contexto de mayor igualdad de género dentro del hogar. De hecho, anteriomente ya se observó que la reducción del número de horas dedicadas por parte de las mujeres españolas a las tareas dentro del hogar no implica que los hombres dedicaran más tiempo a estas tareas (Domínguez-Folgueras 2012). Además, el sector privado de servicios domésticos está compuesto de manera masiva por mujeres.

Por otro lado, en el caso masculino se observan diferencias significativas en su nivel de satisfacción en relación con su contribución relativa al presupuesto familiar. Aquellos que declaran ser los que más contribuyen al total de los ingresos familiares muestran mayores niveles de satisfacción respecto de los que declaran contribuir menos que sus parejas, siempre en el contexto de los hogares con dificultades económicas. Por tanto, parece que el hecho de ser el proveedor principal de resursos materiales dentro del hogar está asociado con los valores de satisfacción en este grupo de hombres, mientras que entre aquellos que declaran no tener dificultades económicas, el hecho de ser los máximos proveedores de recursos económicos no parece dar un impulso extra a sus niveles de satisfacción. Lo mismo ocurre en el caso de la participación en las tareas del hogar, en las que únicamente se observan diferencias significativas de nuevo en los hombres con dificultades económicas, entre los cuales se demuestra que dedicar menos tiempo que sus parejas a las tareas del hogar es una situación asociada a mayores niveles de satisfacción. Consecuentemente, al contrario que en el caso femenino, la satisfacción con la vida de los hombres españoles adultos parece determinarse, en mayor medida, a partir de su situación individual, más allá de las características del conjunto del hogar, lo cual apuntaría a que, en este grupo concreto de hombres, su posición dentro de la esfera pública sigue marcando su percepción sobre la satisfacción con la vida.

Por tanto, nuestro trabajo apunta a que en España se pueden observar dos situaciones diferentes en cuanto al efecto de los comportamientos de género sobre la satisfacción con la vida. Cuando no se declara tener dificultades económicas, parece que los comportamientos de género que se observan en el ámbito del hogar tienen una menor influencia sobre los niveles de satisfacción con la vida por parte de los individuos, sean mujeres u hombres. Sin embargo, los hogares que declaran tener dificultades eco- 
nómicas muestran un perfil de género mucho más cercano al tradicional. De este modo, el contexto económico del hogar funcionaría como catalizador de la mayor o menor asociación de las diferentes situaciones de desigualdad de género dentro del hogar y con la satisfacción con la vida de los individuos. Un contexto económico adverso reduciría la capacidad de negociación de aquella parte con un potencial menor dentro del mercado de trabajo remunerado, que desafortunadamente sigue siendo la mujer (Vicent et al. 2013). A pesar de que la primera etapa de la revolución de género se centró en la esfera pública, el mercado laboral español sigue situando a las mujeres en desventaja respecto a los hombres en cuanto a sus posibilidades de encontrar y mantener un trabajo o, incluso, en los niveles salariales a igualdad de tareas y responsabilidades (Villar 2010).

Pero, además, si analizaramos la relación en el sentido contrario, podríamos entender que los roles de género más tradicionales se asocian con una mayor propensión a que los hogares presenten una situación de dificultad económica. Teniendo en cuenta el progresivo deterioro de las condiciones laborales y salariales de la gran mayoría de la población activa como consecuencia de las reformas laborales iniciadas en el contexto de la reciente crisis económica (Medina et al. 2012), preservar el modelo tradicional de especialización dentro de la pareja, en el cual un único miembro participa en el mercado laboral, puede ser visto como un lujo al alcance de un porcentaje cada vez menor de la población. De forma complementaria, se podría apuntar al hecho de la transmision intergeneracional tanto de los comportamientos de género como de las desigualdades sociales (Minguez 2011; Billari y Liefbroer 2007), por

\section{Bibliografía}

Aassve, A., G. Fuochi y L. Mencarini. 2014. "Desperate housework: Relative resources, time availability, economic dependency and gender ideology across Europe". Journal of Family Issues 35(8): 1000-1022. https://doi. org/10.1177/0192513X14522248.

Ajenjo, M. y J. García. 2014. "Cambios en el uso del tiempo de las parejas. ¿Estamos en el camino hacia una mayor igualdad?". Revista Internacional de Sociología 72.2: 453-476. https://doi.org/10.3989/ris.2012.05.28.

Alonso-Pérez, M. y E. Furió-Blasco. 2007. "El papel de la mujer en la sociedad española”. Universidad de Valencia, Economía Aplicada - Grup d'Investigació - Economia, treball i territorio, 19.

Appelbaum, E. y R. Milkman, R. 2013. Unfinished Business. Ithaca, NY: Cornell University Press.

Arpino, B., G. Esping-Andersen y Pessin, L. 2015. "How do changes in gender role attitudes towards female employment influence fertility? A macro-level analysis". European Sociological Review 31: 370-382. https:// doi.org/10.1093/esr/jcv002.

Arpino, B. y H. de Valk. 2018. "Comparing life satisfaction of immigrants and natives across Europe: the role of social lo que la evolución de ambos factores podría ir en paralelo desde una perspectiva generacional $\mathrm{y}$, por tanto, los cambios en el caso de las personas con un origen social más bajo serían mucho más lentos. Esta bidireccionalidad entre los valores de género y la situación económica se debería testar de manera específica en el futuro.

Nuestro trabajo no está exento de limitaciones. La naturaleza transversal de los datos analizados no permite ir más allá de resultados sobre las asociaciones entre variables, sin poder conjeturar acerca de los posibles efectos causales. Además, el reducido número de casos en las muestras españolas de las dos ediciones de la ESS analizadas han dificultado la obtención de resultados estadísticamente significativos. Esta limitación se redujo al analizar las ediciones de 2004 y 2010, después de comprobar que el año no ejercía un efecto significativo sobre los resultados. Sin embargo, precisamente la falta de diferencia entre años no ha permitido evaluar la posible evolución en el tiempo de las interacciones entre nuestras variables.

En un futuro se plantea la posibilidad de ampliar los análisis a todos los países disponibles en la ESS que hayan participado en las ediciones de 2004 y 2010 para, de este modo, explorar los posibles patrones diferentes en cuanto a nuestro objeto de estudio en todo el contienente. Además, siguiendo en el caso concreto de España, se planea analizar con mayor detalle los mecanismos que se encuentran detrás de los resultados obtenidos, yendo de este modo más allá del marco de referencia proporcionado por la revolución de género, hacia el conocimiento de las razones que explican el proceso de cambio en las dos etapas del caso español.

contacts". Social indicators research 137(3): 1163-1184. https://doi.org/10.1007/s11205-017-1629-x.

Billari, F. C. y A. C. Liefbroer. 2007. "Should I Stay or Should I Go? The Impact of Age Norms on Leaving Home". Demography 44 (1): 181-198. https://doi.org/10.1353/ dem.2007.0000.

Borràs, V., M. Ajenjo y S. Moreno-Colom. 2018. "More time parenting in Spain: a possible change towards gender equality?". Journal of Family Studies 1-16. https://doi. org/10.1080/13229400.2018.1440618.

Brinton, M. y D. J. Lee. 2013. Gender essentialism and low fertility in postindustrial societies. Population and Development Review.

Bueno, X. y M. C. Brinton. 2019. "Gender egalitarianism, perceived economic insecurity, and fertility intentions in Spain: A qualitative analysis". Population studies 73(2), 247-260.

Cherlin, A. J. 2016. "A happy ending to a half-century of family change?". Population and Development Review 42(1), 121-129. https://doi.org/10.1111/j.17284457.2016.00111.x.

Doblhammer, G. y J. Gumà. 2018. "Gender differences in the relationship between household position and health in twelve European countries: are they associated with 
the value climate?" en $A$ demographic perspective on gender, family and health in Europe. Cham, Switzerland: Springer. https://doi.org/10.1007/978-3-31972356-3_6.

Domínguez-Folgueras, M. 2012. "La división del trabajo doméstico en las parejas españolas. Un análisis de uso del tiempo". Revista Internacional de Sociología 70(1), 153-179. https://doi.org/10.3989/ris.2009.08.26.

England, P. 2010. "The gender revolution: Uneven and stalled". Gender \& Society 24: 149-166. https://doi. org/10.1177/0891243210361475.

Esping-Andersen, G. y F. Billari. 2015. "Re-theorizing family demographics" Population and Development Review 41(1): 1-31 https://doi.org/10.1111/j.1728-4457.2015.00024.x.

EUROSTAT. 2018. "Gender Equality". Consultado el 20 de Enero de 2019. (https://ec.europa.eu/eurostat/web/sdi/ gender-equality).

EUROSTAT. 218. "Labour Force Survey". Consultado 20 de Enero 2019. (https://ec.europa.eu/eurostat/web//fs/ data/database).

Fernández-Alonso, A. M., M. Trabalón-Pastor, C. Vara, P. Chedraui y F. R. Pérez-López. 2012. "Life satisfaction, loneliness and related factors during female midlife". Maturitas 72(1): 88-92. https://doi.org/10.1016/j.maturitas.2012.02.001.

Fitzgerald, R. y R. Jowell. 2010. "Measurement equivalence in comparative surveys: the European Social Survey (ESS) - from design to implementation and beyond". Survey methods in multinational, multiregional, and multicultural contexts 485-495. https://doi. org/10.1002/9780470609927.ch26.

Freedman, E. 2007. No turning back: The history of feminism and the future of women. Nova York: Ballantine Books.

FOESSA. 2019. VIII Informe sobre exclusión y desarrollo social en España. Madrid: Fundación FOESSA.

Gerson, K. 2010. The Unfinished Revolution: How a New Generation Is Reshaping Family,Work, and Gender in America. New York: Oxford University Press.

Gil-Hernández C. J., I. Marqués-Perales y S. Fachelli. 2017. "Intergenerational social mobility in Spain between 1956 and 2011: The role of educational expansion and economic modernisation in a late industrialised country". Research in social stratification and mobility 51, 14-27. https://doi.org/10.1016/j.rssm.2017.06.002.

Goldscheider, F., E. Bernhardt y T. Lappegård. 2015. "The gender revolution: A framework for understanding changing family and demographic behavior". Population and Development Review 41(2): 207-239. https:// doi.org/10.1111/j.1728-4457.2015.00045.x.

Gumà, J., R. Treviño y A. D. Cámara. 2015. "Posición en el hogar y género. Desigualdades en la calidad de vida relacionada con la salud entre la población adulta en España". Revista internacional de sociología 73(1), 003. https://doi.org/10.3989/2013.03.04.

Haas, L. 1992. Equal Parenthood and Social Policy: A Study of Parental Leave in Sweden. Albany, NY: SUNY Press.

Koch, A., V. Halbherr, I. A. Stoop y J. W. Kappelhof. 2014. Assessing ESS sample quality by using external and internal criteria. Mannheim: European Social Survey, GESIS.

Lewis, J., M. Campbell y C. Huerta. 2008. "Patterns of paid and unpaid work in Western Europe: gender, commodification, preferences and the implications for policy" Journal of European Social Policy 18.1: 21-37. https:// doi.org/10.1177/0958928707084450.
Lombardo, E. y M. León. 2014. "Políticas de igualdad de género y sociales en España: origen, desarrollo y desmantelamiento en un contexto de crisis económica". Investigaciones feministas 5: 13-35. https://doi.org/10.5209/ rev_INFE.2014.v5.47986.

Lutz, W. 2010. "Education will be at the heart of 21st century demography". Vienna Yearbook of Population Research 8: 9-16. https://doi.org/10.1553/populationyearbook2010s9.

Martín, R. y N. Sánchez-Sánchez. 2007. “Los diferenciales salariales por género en España durante el desarrollismo franquista". Revista Española de Investigaciones Sociológicas (REIS) 117.1: 143-160. https://doi. org/10.2307/40184756.

Martínez-Celorrio, X. y A. Marín-Saldo. 2012. "Educación y movilidad social en España". en Informe España 2012: Una interpretación de su realidad social. Centro de Estudios del Cambio Social. Madrid: Fundación Encuentro.

Maté, C. y N. Acarín. 2011. "Encuesta sobre la elección de pareja a estudiantes de la Universitat Pompeu Fabra, Barcelona (20 a 27 años)". Summa Psicológica UST 8(1): 37-46. https://doi.org/10.18774/448x.2011.8.80.

Medina, J. A., J. C. Aguilera, L. de la Fuentes Sanz, A. M. Poza, y A. O. Jáuregui. 2012. "Trabajadores pobres y empobrecimiento en España”. Zerbitzuan: Gizarte zerbitzuetarako aldizkaria-Revista de servicios sociales 52: 119-128. https://doi.org/10.5569/1134-7147.52.07.

Mencarini, L. y M. Sironi. 2010. "Happiness, housework and gender inequality in Europe". European Sociological Review 28(2): 203-219. https://doi.org/10.1093/esr/jcq059.

Minguez, A. M. 2011. "La reproducción intergeneracional de las desigualdades educativas: límites y oportunidades de la democracia". Revista de educación 1: 183-206.

Mood, C. 2010. "Logistic regression: Why we cannot do what we think we can do, and what we can do about it". European sociological review 26(1): 67-82. https://doi. org/10.1093/esr/jcp006.

Moreno-Mínguez,A. 2010. "Family and gender roles in Spain from a comparative perspective". European Societies 12(1): 85-111. https://doi.org/10.1080/14616690902890321.

Murillo-Huertas, I.P. y H. J. Simón-Pérez. 2014. "La Gran Recesión y el diferencial salarial por género en España”. Hacienda Pública Española 208:39-76.

OECD. 2018. "Ageing and Employment Policies - Statistics on average effective age of retirement". Consultado el 12 de Enero de 2020. http://www.oecd.org/employment/ emp/average-effective-age-of-retirement.htm.

Oksuzyan, A., K. Juel, J. W. Vaupel, y K. Christensen. 2008. "Men: good health and high mortality. Sex differences in health and aging". Aging clinical and experimental research 20(2): 91-102. https://doi.org/10.1007/ BF03324754.

Orru, K., H. Orru, M. Maasikmets, R. Hendrikson, y M. Ainsaar. 2016. "Well-being and environmental quality: Does pollution affect life satisfaction?". Quality of Life Research 25(3): 699-705. https://doi.org/10.1007/s11136015-1104-6.

Pessin, L. 2018. "Changing gender norms and marriage dynamics in the United States" Journal of Marriage and Family 80(1): 25-41. https://doi.org/10.1111/jomf.12444.

Prieto, R. R. 2010. "Valores sociales y división sexual del trabajo en las familias españolas: permanencias de género y cambio social". Comunicación presentada en el Congreso de la Federación Española de Sociología, 2 de julio, Pamplona (España). 
Rosales, R. M. 2003. Trabajo, salud y sexualidad: las cargas de trabajo laborales y reproductivas en la salud de las mujeres. Barcelona: Icaria Editorial.

Sassler, S. y D. T. Lichter. 2020. "Cohabitation and Marriage: Complexity and Diversity in Union Formation Patterns". Journal of Marriage and Family 82(1): 35-61. https:// doi.org/10.1111/jomf.12617.

Sayer, L. C. 2015. "Trends in women's and men's time use, 1965-2012: Back to the future?," en Susan M. McHale, Valarie King, Jennifer Van Hook, and Alan Booth (eds.), Gender and Couple Relationships. Dordrecht: Springer. https://doi.org/10.1007/978-3-319-21635-5_2.

Stanfors, M. y F. Goldscheider. 2017. "The forest and the trees: Industrialization, demographic change, and the ongoing gender revolution in Sweden and the United States, 1870-2010". Demographic Research 36, 173226. https://doi.org/10.4054/DemRes.2017.36.6.
Valiente-Fernández, C. 1997. “¿ Algo mas que” ganadores del pan"?: El papel de los hombres en el ámbito familiar en España (1975-1996)". Revista Española de Investigaciones Sociológicas (REIS) 221-243. https://doi. org/10.2307/40184014.

Van der Meer, P. H. 2014. "Gender, unemployment and subjective well-being: Why being unemployed is worse for men than for women". Social Indicators Research 115(1): 2344. https://doi.org/10.1007/s11205-012-0207-5.

Vicent, L., Castro, C., Agenjo, A., Herrero, Y. y Herrero, S. F. 2013. El desigual impacto de la crisis sobre las mujeres. Madrid. Fuhem.

Villar, A. 2010. Mujeres y mercado laboral en España: cuatro estudios sobre la discriminación salarial y la segregación laboral. Madrid: Fundacion BBVA.

Tabla A1.

Características de la muestra de trabajo según la edición de la ESS

\begin{tabular}{|c|c|c|c|}
\hline & & 2004 & 2010 \\
\hline \multirow{4}{*}{ Capacidad económica del hogar } & Cómodamente & $40,3 \%$ & $32,1 \%$ \\
\hline & Llega a final de mes & $42,9 \%$ & $43,6 \%$ \\
\hline & Dificultades & $16,8 \%$ & $24,3 \%$ \\
\hline & Total & $100 \%$ & $100 \%$ \\
\hline \multirow{4}{*}{ Contribución relativa a la economía del hogar } & Menos de la mitad & $38,6 \%$ & $36,2 \%$ \\
\hline & Mitad & $20,1 \%$ & $21,1 \%$ \\
\hline & Más de la mitad & $41,3 \%$ & $42,7 \%$ \\
\hline & Total & $100 \%$ & $100 \%$ \\
\hline \multirow{4}{*}{ Contribución relativa a la realización de las tareas del hogar } & Menos que la pareja & $40,2 \%$ & $42,6 \%$ \\
\hline & Igual que la pareja & $16,2 \%$ & $13,7 \%$ \\
\hline & Más que la pareja & $43,6 \%$ & $43,7 \%$ \\
\hline & Total & $100 \%$ & $100 \%$ \\
\hline \multirow{3}{*}{ Salud } & Buena o muy buena & $67,6 \%$ & $70,5 \%$ \\
\hline & $\begin{array}{l}\text { Regular, mala o muy } \\
\text { mala }\end{array}$ & $32,4 \%$ & $29,5 \%$ \\
\hline & Total & $100 \%$ & $100 \%$ \\
\hline \multirow{4}{*}{ Nivel educativo } & Bajo & $52,9 \%$ & $49,7 \%$ \\
\hline & Medio & $23,3 \%$ & $22,9 \%$ \\
\hline & Alto & $23,8 \%$ & $27,4 \%$ \\
\hline & Total & $100 \%$ & $100 \%$ \\
\hline \multicolumn{2}{|l|}{ Edad media } & 42,4 & 43,3 \\
\hline \multicolumn{2}{|l|}{ Satisfacción con la vida } & 7,4 & 7,4 \\
\hline \multicolumn{2}{|l|}{$\mathbf{N}$} & 660 & 780 \\
\hline
\end{tabular}


Tabla A2.

Coeficientes modelos de regresión lineal según la interacción entre la capacidad económica del hogar y la contribución relativa a los ingresos del hogar

Interacción capacidad

económica y contribución

ingresos hogar (ref:

Cómodamente\#Más mitad)

\begin{tabular}{|c|c|c|c|c|c|c|}
\hline & \multicolumn{3}{|c|}{ Mujeres } & \multicolumn{3}{|c|}{ Hombres } \\
\hline & Coef. & Std. Err. & Sign. & Coef. & Std. Err. & Sign. \\
\hline $\begin{array}{l}\text { Cómodamente\#Menos } \\
\text { mitad }\end{array}$ & 0.09 & 0.34 & & -0.17 & 0.34 & \\
\hline Cómodamente\#Mitad & -0.01 & 0.37 & & 0.09 & 0.24 & \\
\hline $\begin{array}{l}\text { Llega para vivir\#Menos } \\
\text { mitad }\end{array}$ & -0.22 & 0.34 & & -0.73 & 0.31 & * \\
\hline Llega para vivir\#Mitad & -0.49 & 0.37 & & -0.33 & 0.23 & \\
\hline $\begin{array}{l}\text { Llega para vivir\#Más } \\
\text { mitad }\end{array}$ & -0.55 & 0.43 & & -0.25 & 0.15 & \\
\hline $\begin{array}{l}\text { Dificultades\#Menos } \\
\text { mitad }\end{array}$ & -0.93 & 0.36 & ** & -2.12 & 0.39 & *** \\
\hline Dificultades\#Mitad & -0.93 & 0.48 & * & -1.06 & 0.36 & ** \\
\hline Dificultades\#Más mitad & -1.25 & 0.51 & * & -1.04 & 0.20 & *** \\
\hline & -0.03 & 0.01 & *** & 0.01 & 0.01 & \\
\hline 2010 & 0.05 & 0.13 & & 0.15 & 0.12 & \\
\hline $\begin{array}{l}\text { Regular, mala o muy } \\
\text { mala }\end{array}$ & -0.41 & 0.14 & ** & -0.31 & 0.13 & * \\
\hline Medio & -0.23 & 0.17 & & -0.01 & 0.15 & \\
\hline Alto & -0.20 & 0.18 & & 0.05 & 0.15 & \\
\hline Constante & 9.17 & 0.47 & $* * *$ & 7.49 & 0.33 & $* * *$ \\
\hline & 0.08 & & & 0.10 & & \\
\hline & 763 & & & 677 & & \\
\hline
\end{tabular}

Significación: *** $<0,001 ;{ }^{* *}<0,01 ;{ }^{*}<0,05 ; \dagger<0,1$

Tabla A3.

Coeficientes modelos de regresión lineal según la interacción entre la capacidad económica del hogar y la contribución a la realización de las tareas del hogar

\begin{tabular}{|c|c|c|c|c|c|c|c|}
\hline & & \multicolumn{3}{|c|}{ Mujeres } & \multicolumn{3}{|c|}{ Hombres } \\
\hline & & Coef. & Std. Err. & Sign. & Coef. & Std. Err. & Sign. \\
\hline \multirow{8}{*}{$\begin{array}{l}\text { Interacción capacidad } \\
\text { económica y contribución } \\
\text { tareas del hogar (ref: } \\
\text { Cómodamente\#Más) }\end{array}$} & Cómodamente\#Menos & -0.65 & 0.41 & & -0.09 & 0.38 & \\
\hline & Cómodamente\# & -0.26 & 0.27 & & 0.04 & 0.44 & \\
\hline & Llega para vivir\#Menos & -0.97 & 0.38 & * & -0.33 & 0.39 & \\
\hline & Llega para vivir\# & -0.30 & 0.31 & & -0.24 & 0.45 & \\
\hline & Llega para vivir\#Más & -0.44 & 0.17 & ** & -0.94 & 0.47 & * \\
\hline & Dificultades\#Menos & -0.64 & 0.55 & & -1.06 & 0.41 & * \\
\hline & Dificultades\# & -1.08 & 0.43 & * & -2.54 & 0.50 & *** \\
\hline & Dificultades\#Más & -1.13 & 0.21 & $* * *$ & -1.33 & 0.57 & * \\
\hline Edad & & -0.03 & 0.01 & *** & 0.01 & 0.01 & \\
\hline Año (ref: 2004) & 2010 & 0.06 & 0.13 & & 0.12 & 0.12 & \\
\hline Salud (ref: Buena o muy buena) & $\begin{array}{l}\text { Regular, mala o muy } \\
\text { mala }\end{array}$ & -0.40 & 0.14 & ** & -0.30 & 0.13 & * \\
\hline \multirow{3}{*}{ Nivel educativo (ref: bajo) } & Medio & -0.28 & 0.17 & $\dagger$ & -0.01 & 0.15 & \\
\hline & Alto & -0.24 & 0.17 & & 0.09 & 0.15 & \\
\hline & Constante & 9.38 & 0.37 & *** & 7.47 & 0.48 & $* \star \star *$ \\
\hline $\mathbf{R}^{2}$ & & 0.09 & & & 0.12 & & \\
\hline $\mathbf{N}$ & & 763 & & & 677 & & \\
\hline
\end{tabular}

Significación: ${ }^{* * *}<0,001 ;{ }^{* *}<0,01 ;{ }^{*}<0,05 ; \dagger<0,1$ 
JORDI GUMÀ es investigador del Departamento de Ciéncias Políticas y Sociales de la Universidad Pompeu Fabra e IP del proyecto "Prevention is better than cure when ageing is behind the door: interplay between social determinants of health in Spain (INTERSOC-HEALTH)" (RTI2018-099875-J-I00-MCIU/AEI/FEDER, UE) financiado por el FEDER-Ministerio Español de Ciencia, Innovación y Universidad-Agencia Española de Investigación. Su investigación se centra en los determinantes sociales de la salud desde una perspectiva de género.

BRUNO ARPINO es profesor del Departamento de Estadística, Informática, Aplicaciones de la Universidad de Florencia. Su investigación se centra en la demografía social, la estadística inferencial y la estadística social. 